\title{
Second-order non-local effects mitigation in BOTDA sensors by tracking the BFS profile
}

\author{
Juan José Mompó, Haritz Iribas, Javier Urricelqui, and Alayn Loayssa* \\ Departamento de Ingeniería Eléctrica y Electrónica, Universidad Pública de Navarra, Campus \\ de Arrosadía s/n, 31006, Pamplona, Spain
}

\begin{abstract}
We demonstrate a technique to mitigate the residual second-order non-local effects in Brillouin optical time-domain analysis (BOTDA) sensors in which the Brillouin frequency shift (BFS) profile is not uniform along the fiber. It is based on adding a wavelength modulation to the probe wave that makes it track the average BFS found along its way. Using this method we are able to inject a total probe wave power of $15 \mathrm{dBm}$ in a $120-\mathrm{km}$ sensing fiber link, which, to the best of our knowledge, is the highest probe power ever demonstrated in a long-range BOTDA sensing fiber link. The enhancement in the detected signal-to-noise ratio brought by the use of such power provides $2-\mathrm{MHz}$ BFS measurement precision at the end of the $120-\mathrm{km}$ sensing link with $3-\mathrm{m}$ spatial resolution, all without the need to resort to additional means such as the use of coding or Raman gain.
\end{abstract}

Keywords: Stimulated Brillouin scattering, Brillouin optical time-domain analysis, strain and temperature measurement, second-order non-local effects

\section{INTRODUCTION}

The range of Brillouin optical time-domain analysis (BOTDA) sensors is limited by the detected signal-to-noise ratio (SNR). This can be enhanced by increasing the probe power injected into the fiber. However, the maximum probe power is limited by the onset of so-called non-local effects (NLE), which are due to the transfer of energy from the pump pulse to the probe in its interaction along the fiber. ${ }^{1}$ These NLE can be mitigated to first order by deploying a dual-probe BOTDA setup in which the energy transferred from the pump pulse to one probe is compensated by that received from the other. ${ }^{2}$ Nevertheless, this does not solve the problem completely, as it has been found that during the scanning of the Brillouin spectra, when the pump-probe frequency offset is different from the local Brillouin frequency-shift (BFS) of the fiber, the gain and loss spectrum induced by both probe waves on the pump do not perfectly overlap, and hence, the pump pulse experiences a non-flat transfer function that distorts its spectrum. ${ }^{3}$ These second-order NLE limit the maximum probe power that can be deployed to around $-5 \mathrm{dBm}$ in long-length sensing links.

Several approaches have been recently developed to compensate second-order NLE in BOTDA sensors. The first one is a system based on a dual-probe side-band BOTDA where the difference between both probe waves is maintained at twice the BFS of the fiber. ${ }^{4}$ However, this only works if the BFS profile along the fiber is fairly uniform. Another proposal aims to compensate second-order NLE by using a four-tone probe wave. ${ }^{5}$

We have recently proposed another scheme to mitigate second-order NLE that is also based on a dual-probe side-band BOTDA configuration in which the optical frequency of the probe wave is modulated along the fiber. ${ }^{6}$ This generates a net broad gain and loss spectra that overlap over the pump spectrum avoiding first and second order NLE. This configuration has the fundamental advantage compared to previous proposals that the modulation of the frequency of the probe wave additionally serves to increase the effective Brillouin threshold of the fiber.

In this work, we demonstrate a technique to avoid the degradation in performance experienced by methods that compensate second order NLE when the BFS of the fiber is not uniform. This novel technique is based on adding a wavelength modulation to the probe wave that makes it track the average BFS found along its way. Combining this technique with the BOTDA sensor that uses frequency modulation of the probe wave, we are able to inject the highest optical probe power ever demonstrated to our knowledge in a long-range BOTDA sensor.

*E-mail: alayn.loayssa@unavarra.es

25th International Conference on Optical Fiber Sensors, edited by Youngjoo Chung, Wei Jin,

Byoungho Lee, John Canning, Kentaro Nakamura, Libo Yuan, Proc. of SPIE Vol. 10323

103237E · C 2017 SPIE · CCC code: 0277-786X/17/\$18 - doi: 10.1117/12.2265099

Proc. of SPIE Vol. 10323 103237E-1 


\section{DESCRIPTION OF THE TECHNIQUE}

Fig. 1(a) schematically depicts the operation of our previous technique for second-order NLE compensation. The probe waves interact with the pump pulse as in a conventional dual-probe side-band BOTDA configuration. Moreover, the optical frequency of the probes wave is modulated along the fiber with a sawtooth profile, so that the net Brillouin interactions generated by each probe wave on the pump become a broad gain and loss spectra with a bandwidth approximately equal to the peak-to-peak optical frequency deviation of the modulation. ${ }^{6}$ In Fig. 1(a) a fiber with two different BFS along its length is assumed. In the first section the modulation of the optical frequency of the probe is made around a mean value that coincides with the BFS of that section (BFS1). In that case, as it is schematically depicted in the figure, the broad gain and loss spectra perfectly compensate and the pump spectra experiences no distortion. However, on the second section, there is a different BFS (BFS2) that makes the broad gain and loss spectra not to be perfectly centered on the pump pulse optical frequency. This leads to a distortion of the pump pulse spectral flanks.

In order to solve this limitation, brought by the non-uniformity of the BFS of the fiber, Fig. 1(b) depicts the fundamentals of the technique for BFS tracking. The basic idea is to add an optical frequency modulation offset that tracks the changes in the average BFS of the fiber. This is outlined in Fig. 1(b), where an offset in the optical frequency modulation of the probe is introduced so that it compensates the change in average BFS in the second section of the fiber. In this way, the gain and loss spectra induced by both probes are always centered over the pump wave spectrum and they canceled out. Therefore, spectral distortion of pump pulse is avoided. Notice that the BFS tracking does not need to be very precise or very fast. It is necessary just to adapt to changes in the average BFS profile of different sections of the fiber. This can be extracted from the measurements that the BOTDA system is continuously performing or even from previous measurements of the BFS using low spatial resolution.

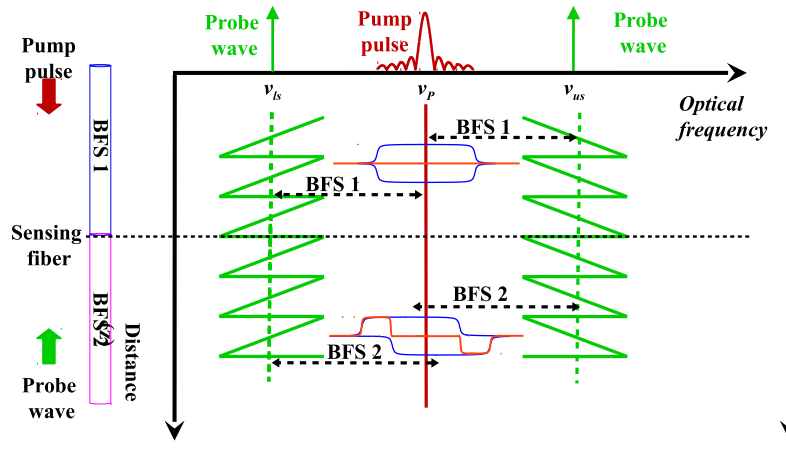

(a)

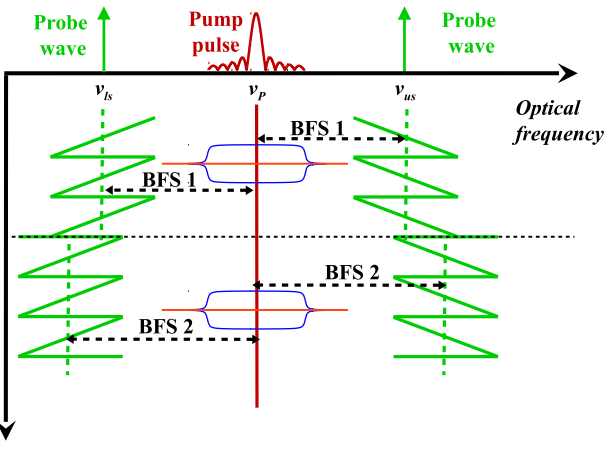

(b)

Figure 1. (a) Spectra of the optical waves present in the optical fiber and (b) Brillouin interaction when BFS is not compensated with the central frequency (c) BFS tracking applied to the central frequency of probe wave.

\section{EXPERIMENTAL RESULTS}

Fig. 2 depicts the setup used in this technique. It is based on a conventional dual-probe side-band BOTDA setup in which the signal generator used to provide the microwave frequency that controls the optical frequency sweep of the probe wave is substituted by an arbitrary waveform generator (AWG). The AWG generates the frequency modulation of the probe wave following a saw-tooth profile and also modifies the average frequency of the probe wave in order to follow the BFS profile of the fiber. Moreover, it also provides the electrical pulse that controls the semiconductor optical amplifier (SOA) switch. In our experiments, the AWG provides a microwave signal with a central frequency of $10.8 \mathrm{GHz}$ and a peak-to-peak frequency deviation of $300 \mathrm{MHz}$ and a period of 12.5 micro-seconds. The peak power of the pump pulse finally injected in the fiber was $80 \mathrm{~mW}(19 \mathrm{dBm})$.

First, we analyzed the effects of BFS non-uniformity on the performance of the technique for second-order NLE compensation that uses modulation of the frequency of the probe wave but without BFS tracking. ${ }^{6}$ We started by characterizing the BFS uniformity of the optical fiber using low spatial resolution. Fig. 3(a) shows 


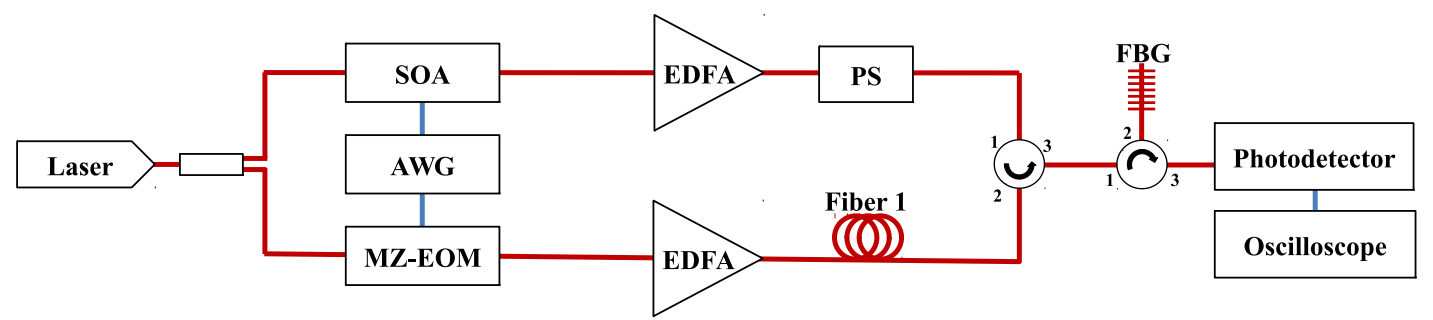

(a)

Figure 2. Experimental setup for the BOTDA sensor based on tracking the BFS with frequency modulation of the probe wave.

the BFS profile along the fiber link. Notice that the link is divided in 3 sections. The first one with a length of $50 \mathrm{~km}$ and a BFS of $10.783 \mathrm{GHz}$, second one of $50 \mathrm{~km}$ and a BFS around $10.767 \mathrm{GHz}$ and the last one, which is inside a climate chamber in order to maintain its temperature stable, with a BFS of $10.813 \mathrm{GHz}$.

Then, we measured the optical frequency response experienced by the pump pulse as a result of its interaction with the probe waves, which is shown in Fig. 3(b) for different probe waves powers. This measurement was performed using the optical single side-band (OSSB) modulation method, ${ }^{7}$ in which the upper branch of the setup in Fig. 2 was substituted by two Mach-Zehnder electro-optic modulator (MZ-EOM), one of them of the dual-electrode type, and an optical filter arranged to generate a OSSB signal whose side-band could be swept over the range of frequencies of the pump pulse and experience the optical transfer function induced by Brillouin interaction with the two probe waves. That transfer function was measured using a vector network analyzer connected to the OSSB modulator, to sweep the frequency of the side-band, and to a wideband receiver located at the far end of the fiber.

Notice that the measurements in Fig. 3(b) reveal that the spectral distortion suffered by the pump wave for low power of the probe waves is negligible. However, as the probe power is increased a non-zero Brillouin interaction distorts the pump spectrum making it asymmetrical in frequency as it gets amplified. Note that the separation between gain and loss peaks that can be observed in Fig. 3(b) is directly related to the peak-to-peak frequency deviation of the probe wave. An analogue result is found when we measure the temporal distortion suffered by the pump pulses after transversing the fiber, as it is shown in Fig. 3(c). Again, for low probe power, the pulses are not distorted and they keep their shape, but for higher power level the pulses loose their shape in the falling edge and a ripple appear on their top making it impossible to perform proper BOTDA measurements. Notice that these results are directly correlated and explained by the measurements in Fig. 3(b).

Finally, we implemented the new technique for BFS tracking by adding an offset to the probe wave modulation that compensated the average BFS variation along the fiber depicted in Fig. 3(a). Fig. 4(b) displays the pulses at the output of the fiber, where it can be seen that distortion is negligible up to $15 \mathrm{dBm}$, the maximum

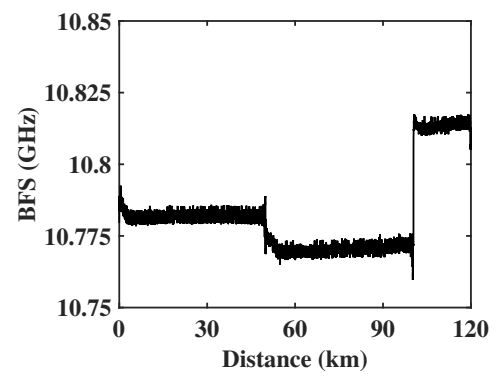

(a)

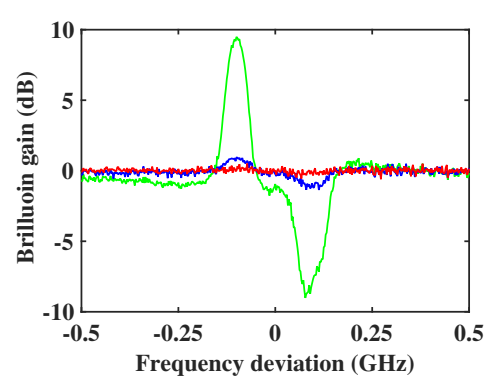

(b)

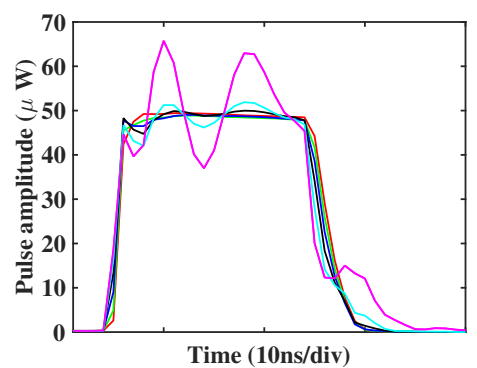

(c)

Figure 3. (a) BFS measured with 200 ns pulse length when BFS tracking is no applied. (b) Frequency response experience by the pump pulse due to the Brillouin interaction with probe waves of different powers: $-3 \mathrm{dBm}(\mathrm{red}), 5 \mathrm{dBm}$ (blue) and $15 \mathrm{dBm}$ (green). (c) $20 \mathrm{~ns}$ pulses at the output of the fiber when probe wave power is $0 \mathrm{dBm}$ (red), $3 \mathrm{dBm}$ (green), $6 \mathrm{dBm}$ (blue), $9 \mathrm{dBm}$ (black), $12 \mathrm{dBm}$ (cyan) and $15 \mathrm{dBm}$ (magenta). 


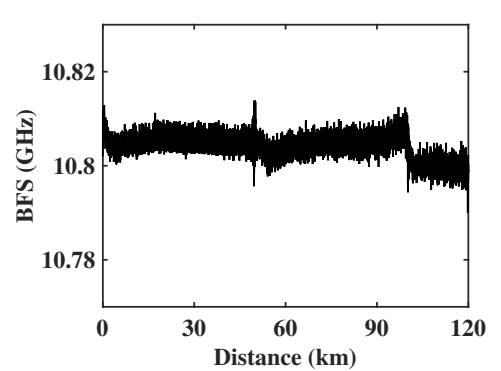

(a)

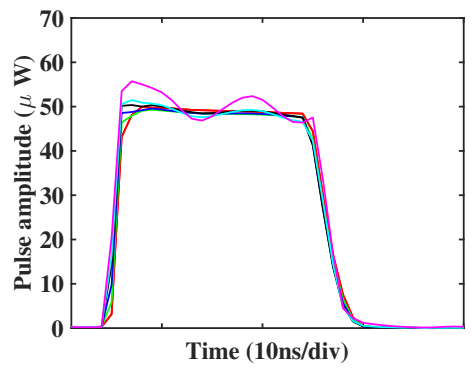

(b)

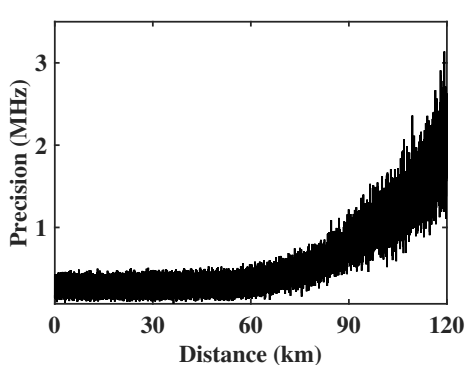

(c)

Figure 4. (a) BFS measured with 30 ns pulse length when BFS tracking is applied. (b) 20 ns pulses at the output of the fiber when probe wave leves is $0 \mathrm{dBm}$ (red), $3 \mathrm{dBm}$ (green), $6 \mathrm{dBm}$ (green), $9 \mathrm{dBm}$ (black), $12 \mathrm{dBm}$ (cyan) and $15 \mathrm{dBm}$ (magenta). (c) Precision of the BFS measurement obtained with probe wave BFS tracked.

power that could be injected in the fiber before the onset of noise induced by spontaneous Brillouin scattering. Fig. 4(a) shows the measured BFS after the offset is added. The real BFS is obtained after substracting the offset from the measurement. Finally, Fig. 4(c) displays the precision of the system that was measured by calculating the standard deviation of a set of 18 consecutive measurements. $2-\mathrm{MHz}$ uncertainty was obtained at the worst-contrast position. A figure of merit ${ }^{1}$ of 1800 has been achieved.

\section{CONCLUSIONS}

The new technique for BFS tracking mitigates second-order NLE and allows to deploy a probe power of $15 \mathrm{dBm}$, which, to the best of our knowledge, is the largest probe power ever demonstrated in a long-range BOTDA setup. The enhancement in the detected SNR brought by the use of such power leads to a performance that has not been previously achieved without resorting to additional means such as the use of coding or Raman gain.

\section{ACKNOWLEDGEMENTS}

The author's wish to acknowledge the financial support from the Spanish Ministerio de Economía y Competitividad through projects TEC2013-47264-C2-2-R and TEC2016-76021-C2-1-R., FEDER funds and the UPNA.

\section{REFERENCES}

[1] Soto, M. A. and Thévenaz, L., "Modeling and evaluating the performance of Brillouin distributed optical fiber sensors," Opt. Express 21, 31347-31366 (Dec 2013).

[2] Minardo, A., Bernini, R., and Zeni, L., "A simple technique for reducing pump depletion in long-range distributed Brillouin fiber sensors," IEEE Sensors Journal 9, 633-634 (June 2009).

[3] Domínguez-López, A., Angulo-Vinuesa, X., López-Gil, A., Martín-López, S., and González-Herráez, M., "Non-local effects in dual-probe-sideband Brillouin optical time domain analysis," Optics Express 23, 10341 (Apr. 2015).

[4] Domínguez-López, A., Yang, Z., Soto, M. A., Angulo-Vinuesa, X., Martín-López, S., Thévenaz, L., and González-Herráez, M., "Novel scanning method for distortion-free BOTDA measurements," Opt. Express 24, 10188-10204 (May 2016).

[5] Hong, X., Lin, W., Yang, Z., Wang, S., and Wu, J., "Brillouin optical time-domain analyzer based on orthogonally-polarized four-tone probe wave," Opt. Express 24, 21046-21058 (Sep 2016).

[6] Ruiz-Lombera, R., Urricelqui, J., Sagues, M., Mirapeix, J., López-Higuera, J. M., and Loayssa, A., "Overcoming non-local effects and Brillouin threshold limitations in Brillouin optical time-domain sensors," IEEE Photonics Journal 7, 1-9 (Dec 2015).

[7] Loayssa, A., Hernández, R., Benito, D., and Galech, S., "Characterization of stimulated Brillouin scattering spectra by use of optical single-sideband modulation," Opt. Lett. 29, 638-640 (Mar 2004). 